\title{
Hemodynamic response to exercise in supine and standing attitudes: an integrated model
}

\author{
Weiwei JIN*, Fuyou LIANG** and Hao LIU*,** \\ * Graduate School of Engineering, Chiba University \\ 1-33, Yayoicho, Inage-ku, Chiba-shi, Chiba 263-8522, Japan \\ E-mail: hliu@faculty.chiba-u.jp \\ **Department of Naval Architecture and Ocean Engineering, Shanghai Jiao Tong University and Chiba University International \\ Cooperative Research Center (SJTU-CU ICRC), Shanghai Jiao Tong University \\ No.800 Dongchuan Road, Minghang District, Shanghai, 200240, China
}

\section{Received 25 September 2015}

\begin{abstract}
Dynamic exercise influences the cardiovascular system in various ways, and the attitude of our body also affect the hemodynamics. In this study, we adopted and integrated a double-closed-loop model consisting of the cardiovascular system (CVS), and the autonomic nervous system (ANS), along with the mathematical models of exercise and body attitude for hemodynamic prediction and cardiovascular regulation. The CVS-ANS-exercise coupling model is validated with two cases of the hemodynamic response to exercises: two different kinds of exercises (one-leg extension and one-leg cycling), and the recovery from one-leg cycling under two different attitudes (supine and standing). The results show that the transition of blood pressure varies during different exercises; the recovery from exercise is more rapid in the standing attitude. We confirmed by comparing simulation results to experimental data, that the simulation model in this study is effective. Furthermore, a simulation-based exercise model can potentially reduce the risk of performing an exercise test in clinical practice.
\end{abstract}

Key words : Cardiovascular system, Autonomic nervous system, Exercise, Attitude effects, One-leg exercise, Recovery from exercises

\section{Introduction}

The cardiovascular system (CVS) is an elaborate material transport network, which prevents the external environment from upsetting the conditions necessary for life within the organism (Claude Bernard (1813-1878)). Appropriate systemic arterial pressure is a key requirement for proper operation of the CVS, and is continuously monitored by various sensors. Whenever the arterial pressure varies from normal conditions, multiple reflex responses are initiated which causes adjustments in cardiac output and total peripheral resistance so as to normalize the arterial pressure. Short-term adjustments (seconds) are accomplished by variations in the activities of the autonomic nervous system (ANS), such as adjusting the heart rate and peripheral vascular resistance. In the long-term (minutes to days), mechanisms such as variations in cardiac output owing to changes in blood volume play an important role in the control of arterial pressure (Mohrman and Heller, (2006)).

With respect to the short-term regulation of arterial pressure, numerous studies have been performed with animals of dogs, cats, and rabbits to identify the roles of the various baroreceptor reflexes (Donald and Edis, (1971); Guo, et al. (1982); Kawada, et al., (2000); Burattini, et al., (2004)). In addition, several mathematical models for baroregulation have also been developed. Sun, et al. (1997) and Liang \& Liu (2006) investigated the relationship between the Valsalva maneuver and the CVS-ANS interactions; Heldt, et al. (2001) and Olufsen et al. (2005) examined the effects that orthostatic stress on the CVS-ANS interactions. However, these models have merely taken into account of the carotid baroreceptor or aortic baroreceptor, or a coupling of both, but most of them did not consider the cardiopulmonary receptor. 
Due to the limitations of experimental conditions and the complicated nonlinear interactions among various regulatory mechanisms associated with exercise, a comprehensive understanding of exercise is still unclear. Li and Bai (2001) investigated the baroregulation in response to moderate dynamic exercise on both simulation and experimental bases with normal and hypertensive groups. Magosso and Ursino (2002) studied the cardiovascular response to dynamic aerobic exercise from rest to moderate exercise with the consideration of central command, arterial baroreflex, lung inflation reflex, and respiratory regulation. In addition, different attitudes produce gravitational-mediated alterations in hemodynamics in terms of pressure and flow rate. The understanding of such influence on exercise and post-exercise cardiovascular functions can provide an insight into the risk factors in cardiovascular diseases (CVD). Takahashi, et al. (2005) performed an experimental study on this topic with a focus on the mechanisms associated with the recovery from cycle exercise, but the mechanism remains unknown yet.

In this study, we aim at establishing an integrated model to investigate the short-term regulation of the CVS, which is applied to investigate the mechanisms of exercise through coupling two systems: the CVS and ANS. In the ANS model, we took into consideration the influences of three receptors, the carotid and aortic baroreceptors, and the cardiopulmonary receptor. With the exercise model, we attempted to inquire how the blood pressure and heart rate react to different exercises (one-leg extension and one-leg cycling). With an attitude model, we simulated the cycling exercise under two different attitudes (supine and standing) to investigate the gravitational-mediated alterations in the CVS as well. We first describe briefly the methodology involving the CVS-ANS coupling model, the attitude model, and the exercise model. We then show a case of the model validation by comparing the simulated results during the rest with reliable experimental data. Furthermore, we present the results of two different one-leg exercises, one-leg extension and one-leg cycling in the supine attitude, and of the recovery from one-leg cycling in two different attitudes, supine and standing.

\section{Methodology}

A double-closed-loop model for hemodynamic prediction and cardiovascular regulation that involves the CVS, ANS, exercise, and body attitude is depicted in Fig.1. Here the CVS and the ANS communicate with each other via blood pressure, heart rate, cardiac contractility, arteriolar resistance, compliance and the unstressed volume of veins. The attitude model adjusts the pressure level in carotid, splanchnic and lower limb, and activates the mechanovasodilation and muscle pump, which affects the blood pressures in the CVS. The exercise model influences the CVS by means of the muscle pump and vasodilation, while interplaying the ANS by resetting the set point of blood pressure level and the summation of the metaboreflex, mechanoreflex and baroreflex.

\subsection{CVS-ANS Coupling Model}

The CVS-ANS coupling model consists of a lumped parameter CVS model and a first-order linear proximal mathematical model for ANS (Liang and Liu (2006)). In this study, the CVS model is a closed loop model, which indicates that there is no need to determine boundary conditions, and only initial conditions (blood flow and velocity) within reasonable ranges were given. In fact, the simulated results (blood flow, pressure, etc) are largely dependent on the physiological parameters, such as the resistance and capacity of the modeled elements, and all the specific values can be found in our previous study (Liang and Liu (2006)). Meanwhile, the ANS model is designed to capture the blood pressure variations in the CVS (carotid, aorta and right atrium) through the afferent pathway; and to substantially regulate the blood pressure by altering the cardiovascular parameters of heart rate, and vascular tone through efferent pathway. Hence, the inputs for the ANS model are the blood pressure variations in the CVS model, and the outputs are the alteration of the cardiovascular parameters in the CVS model. Therefore, the CVS-ANS coupling model is a double-loop model, which is able to perform auto-regulation in the CVS. In addition, the model parameters for the ANS model are the minimal and maximal spikes in the afferent pathway, reference blood pressure in the baroreceptors, minimal and maximal values of the parameters in effectors, fractional factors for baroreceptors, along with time constants, slopes and gains of the equations. The detailed information regarding the parameters can be found in our previous study (Liang and Liu (2006). 


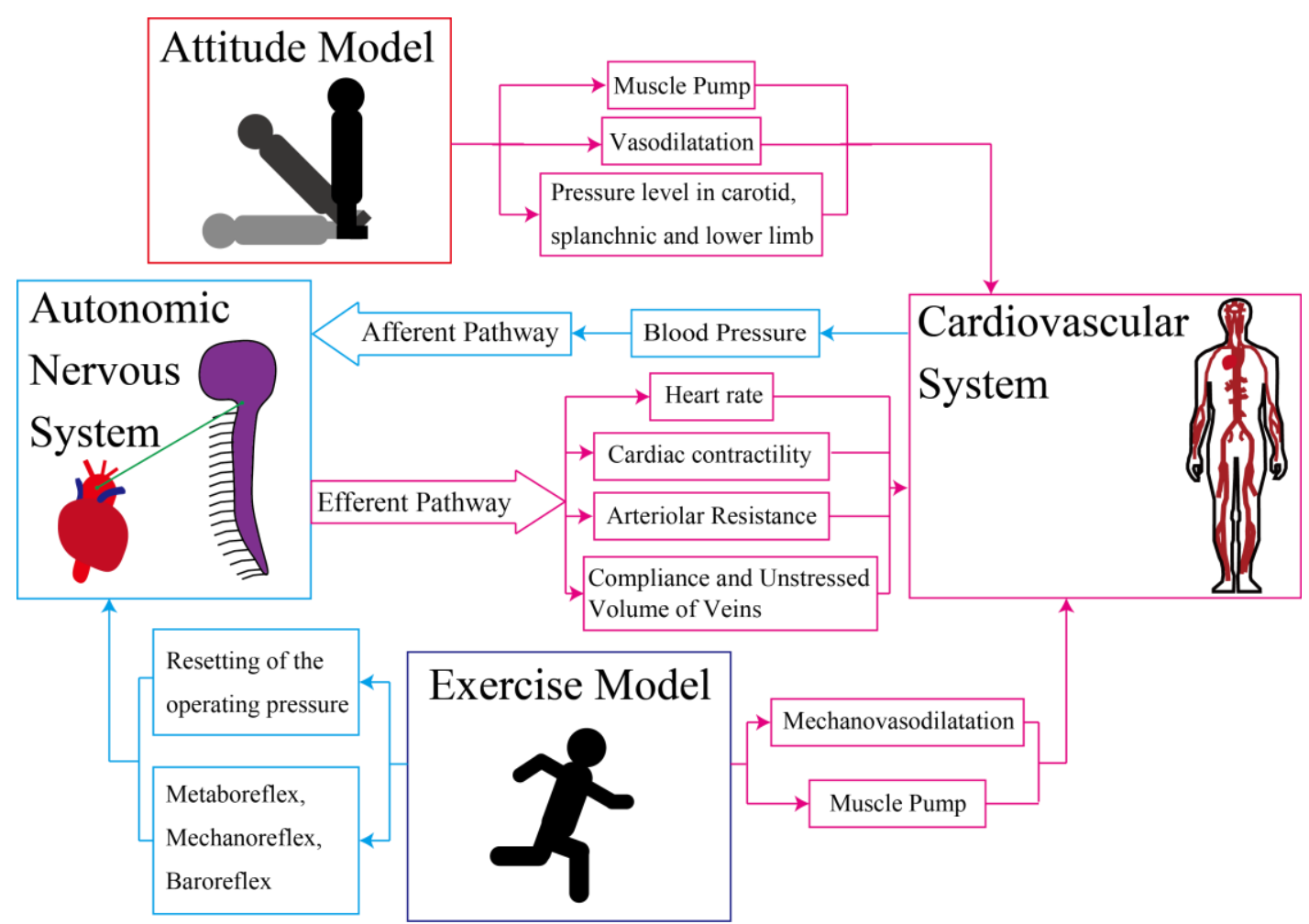

Fig. 1 Schematic description of a double-closed-loop model for hemodynamic prediction and cardiovascular regulation: a CVS model, an ANS model, an attitude model and an exercise model.

\subsection{Attitude Model}

The attitude model contains three parts: the variation of the pressure level in carotid artery, splanchnic artery and lower limb arteries, muscle pump, and mechanovasodilation. The muscle pump in legs and the mechanovasodilation affect the cardiovascular functions in a different way, which is dependent upon the attitudes of a subject (supine or standing). In general, blood pressure level in the carotid artery decreases, while blood pressure level in splanchnic artery and lower limb arteries increases in the standing attitude due to gravity effect. Detailed mathematical description can be found in Appendix A. Based on previous studies (Liang and Liu, 2006; Olufsen, et al. 2005), we reset the pressure levels based on the gravitational effects on hemodynamics with blood pressure at right atrium chosen to be the referential pressure $(+0 \mathrm{mmHg})$. Meanwhile, the muscle pump in legs also alters the blood pressure in lower limb arteries, while the mechanovasodilation affects the resistance in blood vessels. Therefore, input of the attitude model is the body attitude (supine or standing), and the outputs affect the blood pressure and resistance in the modeled blood vessels.

\subsection{Exercise Model}

The exercise model includes the muscle pump model and mechanovasodilation model, which is also incorporated in the attitude model. In addition, the metabovasodilation model, resetting of the operating pressure threshold of arterial baroreflex, central command model, and a summary of mechanoreflex, metaboreflex and baroreflex (Appendix B) are also considered in the exercise model.

Input of the exercise model is oxygen intake relating to the workload, whereas, its outputs extend influence on both the CVS and ANS. The CVS is influenced dynamically by the muscle pump and the metabovasodilation in terms of blood pressure, while the ANS is affected by a combination of the resetting of the operating pressure threshold of arterial baroreflex, central command model, and summary of mechanoreflex, metaboreflex and baroreflex. Here the oxygen intake (workload) is determined based on the previous studies (Ichinose, et al. 2008; Melvin Keller, et al. 2004; Papelier, et al. 1994).

Due to the work of muscle pump, mechano- and metabo- mechanisms vary during the different exercises; for instance, the muscle pump has a prolonging work during the one-leg cycling and only an interstitial work during the one-leg extension. Therefore, we analyzed two different exercises (one-leg extension and one-leg cycling). In addition, 
we validated the simulation results by comparing to the experimental data in the literature (section 3.2).

\section{Results}

\subsection{Attitude Effects}

Gravity has an influence on the venous return during the blood circulation. The pressure level in the portion of the body above the right atria decreases, while the pressure in the portion of the body below the right atria increases. In order to validate this phenomenon, we carried out the simulations on both supine and standing attitudes during rest. The simulation results of blood pressure and heart rate are illustrated in Fig.2 (A) and (B), respectively. From the results, the difference in the time-variation arterial blood pressure (ABP) between the supine and standing attitude is less than 5\% (Fig.2 (A)), whereas the difference in heart rate is more than 15\% (Fig.2 (B)). This indicates that the blood pressure is maintained in a relatively stable state, while the heart rate rises in the standing attitude to ensure a sufficient cardiac output in the circulation system. In the standing attitude, the activation of the muscle pump in legs results in an increase of venous return against gravity, which leads to the slight increasing of blood pressure in the standing attitude. In addition, the minor change in $\mathrm{ABP}$ and the relatively extensive change in heart rate between the supine and standing attitude was also captured by the experiment carried out by Gisolf, et al. in 2005. Since the parameters in the simulation are obtained from a generic population model, while the experimental data are from a specific individul, the difference between the simulation resluts and experimental data appeared. Despite that, the overall tendency for the blood pressure and heart rate between the simulation data and the experimental data are the same. Moreover, to better understand the underline cause of this phenomenon, we calculated stroke volume and cardiac output. The stroke volume in the standing attitude decreases about 25\% compared to that in the supine attitude (Fig. 2 (C)). To compensate the decrease in blood supply, the heart rate in standing attitude is increased. Consequently, despite the decreased stroke volume in standing attitude, the cardiac output maintained in a relatively stable level (Fig. 2 (D)).
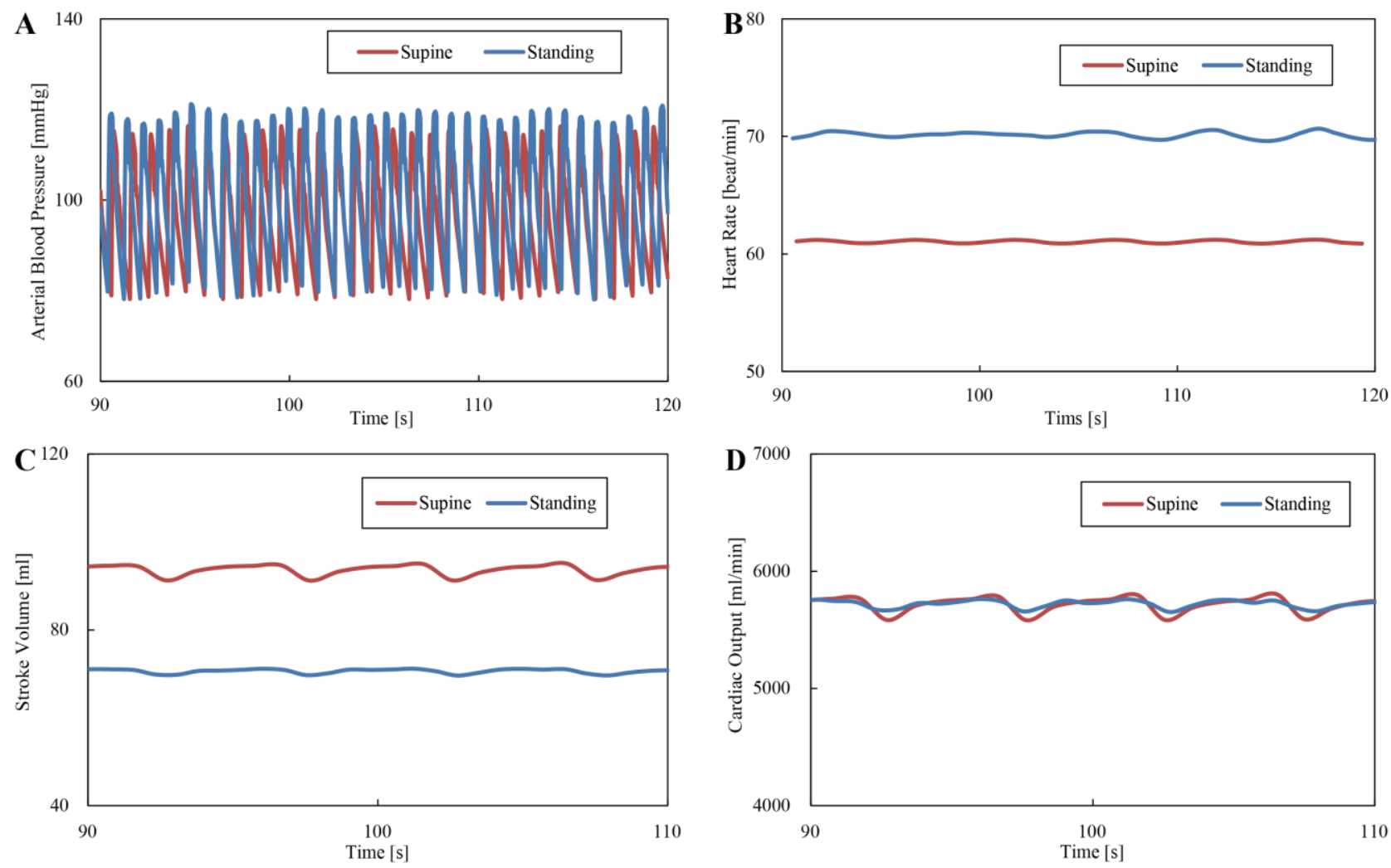

Fig. 2 Simulated ABP (in upper limb) (A), heart rate (B), stroke volume (C), and cardiac output (D) in the supine and the standing attitudes. 

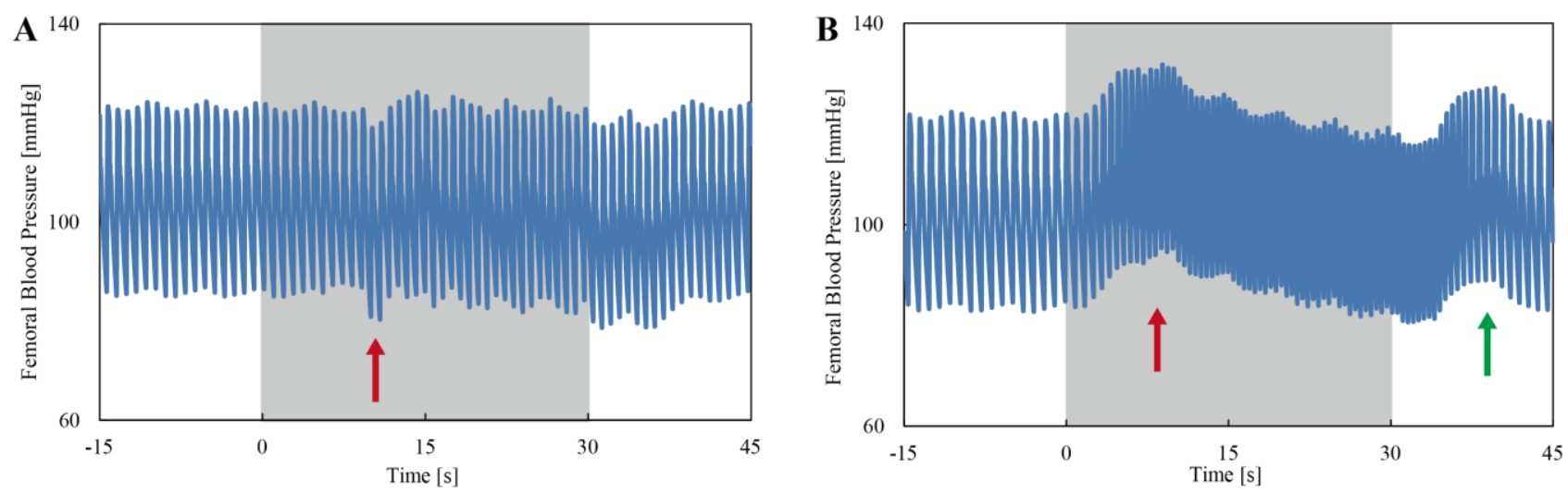

Fig. 3 Simulated femoral blood pressures during one-leg extension (A) and one-leg cycling (B). The exercise start at 0s, and lasts for $30 \mathrm{~s}$.
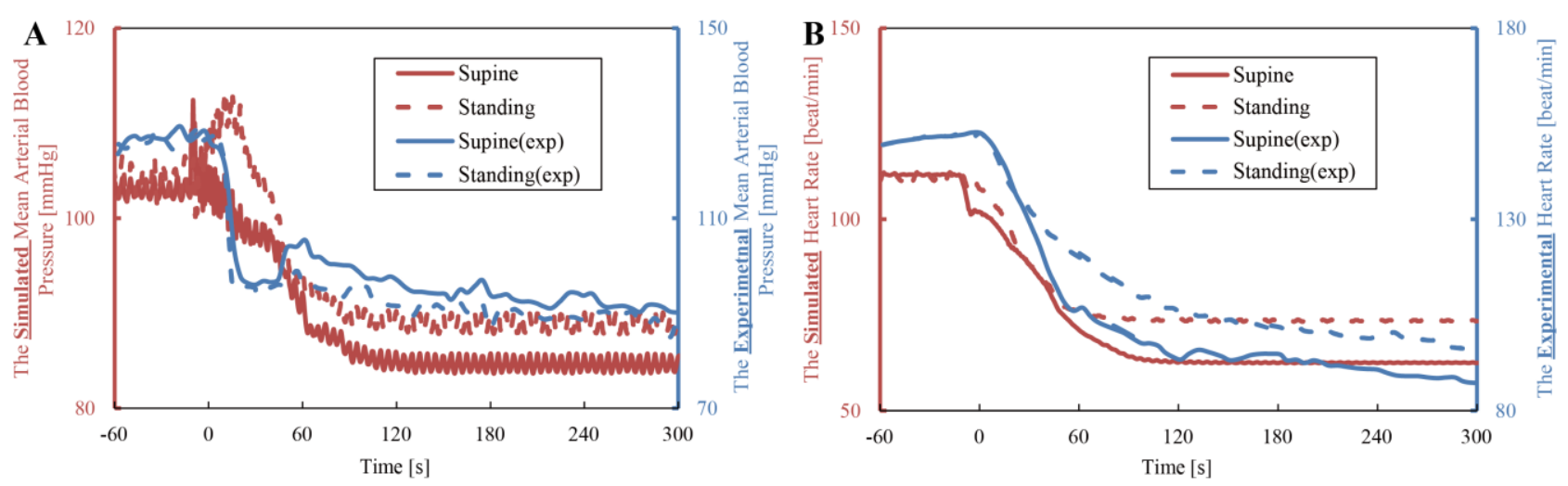

Fig. 4 Comparison of MBP (in upper arm) (A) and heart rate (B) between simulations (the left vertical axis, supine (line), standing (dash line)) and measurements (the right vertical axis, supine (line), standing (dash line)) during the recovery from one-leg cycling in the supine and standing attitudes. The experiment results (measurements) are from Takahashi, et al. 2000. In addition, the subject stopped the exercise at $0 \mathrm{~s}$.

\subsection{Exercise}

In this study, we simulated one-leg extension and one-leg cycling to see the difference in hemodynamics behavior. Furthermore, we simulated the one-leg cycling in the supine and the standing attitude during the recovery from one-leg cycling to reveal underline causes in the exercise brought by the attitude difference.

\subsubsection{One-leg Extension and One-leg Cycling (Supine)}

The work of the muscle pump and mechano- and metabo- mechanisms in our body varies in different exercises. The simulated femoral blood pressure during one-leg extension and one-leg cycling are shown in Fig.3 (A) and (B), respectively. During the early stage of one-leg extension, the fall in blood pressure (red arrow in Fig.3 (A)) is attributable to the rapid increase in vascular conductance in the active leg induced by the mechanovasodilation and muscle pump effect. On the other hand, during the one-leg cycling, the blood pressure had a prolonged decrease due to the continuous work of the muscle pump and mechanovasodilation. Since the parameters in the simulation are obtained from a generic population, while the data in the experiment are individual-specific, the simulated and experimental results show some difference in percentage. Despite that, the blood pressure fall in the early stage of one-leg extension (red arrow in Fig.3 (A)), and the prolonged decrease of the blood pressure during the one-leg cycling (between the red and green arrow in Fig.3 (B)) were observed in the experiment conducted by Radegran, et al., (1998), and Sprangers, et al., (1991), respectively. In addition, the workloads in both cases are mild, which was defined in the previous study (Ichinose, et al. (2008)). The auto-regulation mechanisms is responsible for the recovery of arterial pressure after the initial fall in arterial pressure during one-leg extension, which was also apparent in experimental data (Radegran, et al., (1998)). Meanwhile the autonomic nervous regulation results in an overshoot of blood pressure in the early period of the exercise (the red arrow in Fig.3 (B)), and during the recovery from one-leg cycling (the green arrow in Fig.3 (B)), 
and this damping phenomenon was observed in the experimental data (Sprangers, et al., (1991)) as well.

\subsubsection{Recovery in Supine and Standing Attitude (One-leg Cycling)}

In the previous chapter, it was conformed that different attitudes affect the baseline conditions in hemodynamics, such as blood pressure and heart rate. We assessed how the different attitudes affect recovery from exercise by performing simulation-based one-leg cycling, which is often conducted in clinical practice. The simulation results of mean blood pressure (MBP) and the heart rate recovery from the one-leg cycling are shown in Fig.4 (A) and (B), respectively. In addition, the time duration of the recovery from one-leg cycling is 5 minutes in this simulation. The MBP can be defined in Eq. (1).

$$
M B P=\frac{2}{3} D P+\frac{1}{3} S P
$$

where MBP is the mean blood pressure, DP is the end diastole pressure, and SP is the peak systole pressure, respectively. It shows that the differences of the MBP and the heart rate between the supine and standing attitudes during the one-leg cycling are not significant (less than 5\%). This contributes to the comparatively close vagal nervous and muscle pump effect in different attitude during the exercise. This phenomenon also appeared in the experiment carried out by Takahashi et al. in 2000 (Fig.4). However, due to the difference in the baseline hemodynamic condition in supine and standing, the recovery process in standing is more rapid than that in the supine. After recovering from the exercise, the MBP is slightly high (about 4.5\%) and the heart rate is relatively high (more than 20\%) in standing attitude compared to the supine attitude, which is consistent with the results illustrated in section 3.1. In addition, despite the difference in the maximum and minimum value, the recovery attendance and the difference between supine and standing after recovering from exercise of the simulation results and experiment data (Takahashi et al., 2000) are consistent with each other.

\section{Discussion}

The present study used a coupled model consisting of the CVS, ANS, body attitude, and exercise, where exercise is closely related to the cardiovascular functions and autonomic nervous reactions. A major objective of this study is to integrate the systemic hemodynamic and autonomic control mechanisms that maintain blood pressure homeostasis in human blood circulation with the dynamic effect from exercising. Meanwhile, we also considered the effect on hemodynamic brought by different body attitudes.

\subsection{Two Different One-leg Exercises}

The computational results of two different one-leg exercises indicate that the blood pressure change during the exercise can be different due to the extensiveness of oxygen intake (workload), muscle pump, mechano- and metabovasodilatation, and auto-regulation system. This means that simulations of different exercises can be achieved by applying the underline mechanism, such as the work of muscle pump and vasodilatations, which will allow for a general application for exercise simulation. Furthermore, it is easier to control the onset and offset of the exercise parameters in the simulation base, which means that it can be a useful tool to quantify the underline causes brought by exercise. For instance, further investigation can be done on the distribution of the blood pressure changes brought by the muscle pump and vasodilatations. For clinical applications, the simulation exercise model can be used to design a virtual exercise test for patient with CVD, since the real exercise test sometimes can endanger the patients with CVD (Akinpelu (2015)).

\subsection{Exercise Recovery in Two Different Attitudes}

Gravity on venous blood pooling is also closely related to the cardiovascular functions, which mainly caused by different body attitudes. This affects on the recovery from exercise, which has previously investigated by Takahashi, et al. (2000) on the experimental base. Blood pressure and heart rate in the standing attitude recover more rapidly after exercise than in the standing attitude. In the simulation, the parameter difference between supine and standing mainly contributes to the pressure level, muscle pump and mechanovasodilation, which means those parameters are the 
underline cause for this phenomenon. Further investigation will be needed to determine the distributions of each modeling parameter. The change in blood pressure and heart rate between exercise and rest are severe, and can both be easily obtained in the clinic, which makes those two parameters suitable for patient specific modeling data fitting. The fitting for the blood pressure and heart rate can be achieved by adjusting the parameters in the CVS and ANS. Moreover, by constructing the patient specific model, it is possible to get prediction data that is difficult to obtain with clinical measurements in the simulation bases, such as the blood pressure in the coronary arteries and the cerebral arteries. Furthermore, valve deficiency and diseases related to orthostatic stress are closely associated with the gravity effects; therefore, the model developed in this study can be applied to investigate the risk factors in those diseases. The exercise simulation can help determine the severity of CVD, and evaluating the recover progress for the patient with CVD without endangering them.

\subsection{Limitations}

Despite providing some insights of the exercise mechanism and body attitude effect, there are some limitations. Firstly, some of the results did not agree well with the experimental data. In the simulation results, there was an overshoot in the end of the exercise during the recovery from exercise, while was not seen in the experiments. A possible explanation is that the ANS regulation in the standing attitude is too extensive in the simulation. In addition, the drop in blood pressure is more rapid in the experimental data than that in the simulation results. A possible cause for this is that the decrease in vasodilation was slower in the simulation, since the blood pressure drop is mainly due to the decrease of the total peripheral vascular resistance. Secondly, we only considered some specific elements during exercise. For instance, we did not take time-varying respiration and cellular level mechanisms into account. Thirdly, the computation parameters in this study are from a generic population; individual-specific data needs to be included in order to develop a patient specific model. However, we were still able to reveal some underling mechanisms of how different exercises and exercise in different attitudes affect the CVS.

\section{Conclusion}

In this study, we have established and applied an integrated model by coupling the CVS and ANS to predict hemodynamic reactions during two different exercises (one-leg extension and one-leg cycling) in supine attitudes, recovery from one-leg cycling in two different attitudes (supine and standing). Our results demonstrate that the hemodynamic response in terms of blood pressure varies significantly in different exercises, which also shows distinguished features in different attitudes owing to the gravitation effect. These point to the importance of the CVS-ANS interplay in reasonably predicting the effects of exercise and gravitation on the CVS hemodynamics. The simulation model of exercise and body attitude can be applied to assess the CVD in the clinical field, whereas actual exercise test may endanger the patients (Akinpelu (2015)).

In the future, we will further work on improving the presently coupled model by introducing a more precise muscle pump model and by including the time-varying respiration effect. We will also validate the simulation model for the patients with CVD to develop a virtual exercise test model for clinical application. Furthermore, we will gather individual specific data and tuning for the development of a patient specific model.

\section{Acknowledgement}

The authors would like to thank Dr. Sridhar Ravi for writing advice, Jasmin Wong for English gramma correction, and Dr. Gen Li for discussions. HL was partly supported by Grant-in-Aid for Scientific Research (17300141), JSPS and Research and Development of the Next Generation Integrated Simulation of Living Matter, JST, a part of the Development and Use of the Next Generation Supercomputer Project of the MEXT, Japan. FL was supported in part by the National Natural Science Foundation of China (Grant No. 81370438) and the SJTU Medical-Engineering Cross-cutting Research Project (Grant No. YG2012MS24). Financial support provided by Japanese Government (Monbukagakusho: MEXT) Scholarship to WJ is acknowledged. 


\section{Appendix A}

\section{Attitude Model}

\section{A-1 Change of the pressure level}

We change the pressure level at catrotid artery, splanchnic artery and lower limb (leg) artery during standing up by a sigmoid equation. To capture the transition during standing up, $h$ is defined for the pressure level from gravity as the exponentially increasing function.

$$
h(t)=\frac{h_{M}}{1+e^{-k\left(t-T_{u p}-\delta\right)}},
$$

where $T_{u p}[\mathrm{~s}]$ is the time at which the subject stands up; $h_{M}[\mathrm{~cm}]$ is the maximum height needed for the mean ABP; and $\delta[\mathrm{s}]$ is the latency for the transition to standing (Olufsen, et, al. (2005)).

\section{A-2 Muscle Pump Model}

The following formulae aims to model the contraction mechanisms of muscle pump during the standing and leg exercise. The intramuscular pressure is defined as:

$$
\left\{\begin{array}{lr}
p_{i m}=p_{i m p} \cdot \sin \left(\frac{\pi}{2} \cdot \frac{t_{i m}}{T_{c o n}}\right) & 0 \leq t_{\text {im }} \leq T_{\text {con }} \\
p_{i m}=\left.p_{i m}\right|_{T_{c o n}} \cdot \sin \left(\frac{3 \pi}{2} \cdot \frac{t_{i m}-T_{\text {con }}}{T_{\text {rel }}-T_{\text {con }}}\right) \cdot\left(1-\frac{t_{i m}-T_{\text {con }}}{T_{\text {rel }}-T_{\text {con }}}\right) & T_{\text {con }}<t_{\text {im }} \leq T_{\text {rel }} \\
p_{\text {im }}=0 & \text { without muscle pump }
\end{array}\right.
$$

where $t_{i m}$ [sec] is a moment during muscle contraction-relation cycle; $T_{c o n}$ [sec] is the duration of muscle contraction; $T_{r e l}[\mathrm{sec}]$ is a specified moment before the end of a contraction-relaxation cycle; $p_{i m}[\mathrm{mmHg}]$ is the extravascular pressure; $p_{i m p}[\mathrm{mmHg}]$ is the peak value of $p_{i m}$, which is depend on the oxygen intake (Laughlin et al., (1987)).

\section{A-3 Mechanovasodilation Model}

The following formula aims to model the change of resistance in blood vessels adjusted by mechanovasodilation, which also present sigmoid behavior.

$$
\begin{gathered}
\Delta \sigma_{f r e}=\left(\Delta R_{f r e, m a x}-\Delta R_{f r e, m i n}\right) \cdot \frac{\beta^{f}}{T_{m u s}^{f}+\beta^{f}}+\Delta R_{f r e, m i n}, \\
\frac{d \Delta R\left(t_{\text {mus }}\right)_{f r e}}{d t_{\text {mus }}}=\frac{1}{\tau_{f r e}} \cdot\left[-\Delta R\left(t_{\text {mus }}\right)_{f r e}+\Delta \sigma_{f r e}\right], \\
R\left(t_{\text {mus }}\right)=\lambda_{\text {ref }} \cdot R_{0} \cdot\left(1-\Delta R\left(t_{\text {mus }}\right)_{f r e}\right),
\end{gathered}
$$

where $\Delta R_{\text {fremax }}$ and $\Delta R_{\text {fremin }}$ are the maximal and minimal percent change in resistance due to muscle contraction, respectively; $T_{m u s}[\mathrm{sec}]$ is the duration of muscle contraction-relaxation; $\beta$ is a constant; $f$ is a parameter controlling the slope of the sigmoid; $\tau_{\text {fre }}$ [sec] is a time constant; $\lambda_{\text {ref }}$ is a regulatory parameter derived from autonomic nervous reflexes; $R_{0}$ is the resistance when muscle is in normal resting conditions (Sheriff et al., (1993)). 
Table A Parameters Values

\begin{tabular}{|c|c|c|c|c|c|}
\hline \multicolumn{2}{|c|}{ Chang of the pressure level } & \multicolumn{2}{|c|}{ Muscle Pump model } & \multicolumn{2}{|c|}{ Mechanovasodilation Model } \\
\hline Parameters & Values & Parameters & Values & Parameters & Values \\
\hline$h_{M}$ (carotid) & $20.0[\mathrm{~cm}]$ & $T_{\text {con }}$ & $0.7[\mathrm{sec}]$ & $\Delta \mathrm{R}_{\text {fre,min }}$ & $\begin{array}{l}0.10[\mathrm{mmHg} \\
\left./ \mathrm{ml}^{3}\right]\end{array}$ \\
\hline$h_{M}$ (splanchnic) & $40.0[\mathrm{~cm}]$ & $T_{r e l}$ & $1.0[\mathrm{sec}]$ & $\Delta \mathrm{R}_{\text {fre,max }}$ & $\begin{array}{l}0.65[\mathrm{mmHg} \\
\left./ \mathrm{ml}^{3}\right]\end{array}$ \\
\hline$h_{M}($ lower limb) & $90.0[\mathrm{~cm}]$ & & & $\beta$ & 1.5 \\
\hline$k$ & 2.0 [sec] & & & $\tau_{\text {fre }}$ & $8.0[\mathrm{sec}]$ \\
\hline$\delta$ & $0.4[\mathrm{sec}]$ & & & $f$ & 3.0 \\
\hline
\end{tabular}

\section{Appendix B}

\section{Exercise Model}

\section{B-1 Metabovasodilation Model}

The following formula aims to model the change of the resistance in blood vessels adjusted by metabovasodilation, which also present sigmoid behavior.

$$
\begin{aligned}
& \text { if } \begin{aligned}
& t_{\text {mus }} \leq D_{\text {rat }}, \\
& \Delta R\left(t_{\text {mus }}\right)_{\text {rat }}=0,
\end{aligned}
\end{aligned}
$$

else,

$$
\Delta \sigma_{\text {rat }}=\left(\Delta R_{\text {rat }, \text { max }}-\Delta R_{\text {rat }, \text { min }}\right) \cdot \frac{I_{\text {mus }}^{n}}{\gamma^{n}+I_{\text {mus }}^{n}}+\Delta R_{\text {rat }, \text { min }}
$$

where $\mathrm{I}_{\text {mus }}=\frac{\omega \cdot \mathrm{p}_{\text {imp }}}{T_{\text {fre }}}$,

$$
\begin{aligned}
& \frac{d \Delta R\left(t_{\text {mus }}\right)_{\text {rat }}}{d t_{\text {mus }}}=\frac{1}{\tau_{\text {rat }}} \cdot\left[-\Delta R\left(t_{\text {mus }}\right)_{\text {fre }}+\Delta \sigma_{\text {rat }}\right], \\
& R\left(t_{\text {mus }}\right)=\lambda_{\text {ref }} \cdot R_{0} \cdot\left[1-\left(\Delta R\left(t_{\text {mus }}\right)_{\text {fre }}+\Delta R\left(t_{\text {mus }}\right)_{\text {rat }}\right)\right],
\end{aligned}
$$

where $D_{\text {rat }}[\mathrm{sec}]$ is the delay to the onset of metabolic vasodilatation; $\Delta R_{\text {rat,max }}$ and $\Delta R_{\text {rat,min }}$ are the maximal and minimal percent change in resistance related work rate, respectively; $I_{m u s}\left[\mathrm{mmHg} \cdot \mathrm{ml} \cdot \mathrm{sec}^{-1}\right]$ is the work rate; $\gamma$ $\left[\mathrm{mmHg} \cdot \mathrm{ml} \cdot \mathrm{sec}^{-1}\right]$ and $\omega[\mathrm{ml}]$ are two constants; $\mathrm{n}$ is a parameter controlling the slope of the sigmoid; $\tau_{\text {rat }}$ is a time constant (Ichinose and Nishiyasu, (2005)).

\section{B-2 Model of Resetting of Operating Pressure Threshold of Arterial Baroreflex}

The following formula aims to model the resetting of the operating pressure threshold of arterial baroreflex, which elevates during exercise.

$$
\begin{aligned}
& \Delta p_{\text {exe }}=\Delta p_{\text {exe } \max } \frac{I_{\text {mus }}^{m}}{\phi^{m}+I_{m u s}^{m}}, \\
& p_{\text {n,exe }}=p_{n}+\Delta p_{\text {exe }}
\end{aligned}
$$


where $\Delta p_{\text {exe }}[\mathrm{mmHg}]$ is the offset of the operating pressure threshold; $\Delta p_{\text {exe, max }}[\mathrm{mmHg}]$ is the maximal value for $\Delta p_{\text {exe }} ; \phi\left[\mathrm{mmHg} \cdot \mathrm{ml} \cdot \mathrm{sec}^{-1}\right]$ is a constant; $p_{n \text {,exe }}[\mathrm{mmHg}]$ is a new operating pressure threshold after onset of exercise (Bevegård and Shepherd (1966); Potts et al., (1993); Norton, et al., (1999); Papelier, et al., (1994)).

\section{B-3 Central Command Model}

The following formula aims to model the vagal withdrawal of central command during the exercise, which also present sigmoid behavior. Mathematical functions of vagal withdrawal can be expressed as:

$$
\begin{aligned}
& \Delta F_{\text {vag }}=\frac{\Delta f_{\text {vag,max }} \cdot I_{\text {mus }}}{I_{\text {mus }, \text { max }}}, \\
& \frac{d \Delta f\left(t_{m u s}\right)_{v a g}}{d t_{m u s}}=\frac{1}{\tau_{v a g}} \cdot\left[-\Delta f\left(t_{m u s}\right)_{v a g}+\Delta F_{v a g}\right] \\
& f_{e f p}=F_{a o r p} \cdot \frac{f_{e f p, S}+f_{e f p, E} \cdot \exp \left(\frac{f_{a f, a o r}-f_{a f, a o r, 0}}{k_{e p}}\right)}{1+f_{e f p, E} \cdot \exp \left(\frac{f_{a f, a o r}-f_{a f, a o r, 0}}{k_{e p}}\right)}+F_{c a r p} \\
& \cdot \frac{f_{e f p, S}+f_{e f p, E} \cdot \exp \left(\frac{f_{a f, c a r}-f_{a f, c a r, 0}}{k_{e p}}\right)}{1+f_{e f p, E} \cdot \exp \left(\frac{f_{a f, c a r}-f_{a f, c a r, 0}}{k_{e p}}\right)}-\Delta f\left(t_{m u s}\right)_{v a g},
\end{aligned}
$$

where $I_{\text {mus,max }}\left[\mathrm{mmHg} \cdot \mathrm{ml} \cdot \mathrm{sec}^{-1}\right]$ is a constant indicating the maximal $I_{\text {mus }} ; \Delta f_{\text {vag,max }}[\mathrm{Hz}]$ is the maximal value of vagal withdrawal; $\tau_{v a g}[\mathrm{sec}]$ is a time constant.

\section{B-4 Summation of Mechanoreflex, Metaboreflex and Baroreflex}

The following formula aims to model the combined baroreflex during the exercise, in which the nervous firing is different from the rest state. Mechanoreflex:

$$
\Delta f_{m e c}=k_{m e c} \cdot p_{\text {imp }}+\phi_{\text {mec }}
$$

Where $\Delta f_{\text {mec }}[\mathrm{Hz}]$ is sympathetic efferent discharge elicited by muscle mechanoreceptor; $k_{\text {mec }}[\mathrm{Hz} / \mathrm{mmHg}]$ and $\phi_{m e c}[\mathrm{~Hz}]$ represent slope and intercept, respectively (Yamamoto, et al., (2005)).

Metaboreflex:

If $t_{m u s} \leq D_{m e t}$,

$$
\Delta f\left(t_{m u s}\right)_{m e t}=0 \text {, }
$$

else,

$$
\Delta f\left(t_{m u s}\right)_{m e t}=k_{m e t} \cdot I_{m u s}+\phi_{m e t}
$$

where $D_{\text {met }}$ [sec] is the estimated delay to the onset of metaboreflex; $\Delta f\left(t_{\text {mus }}\right)_{\text {met }}[\mathrm{Hz}]$ is the afferent signal elicited by muscle metaboreceptor; $k_{m e t}$ and $\phi_{m e t}$ represent slope and intercept, respectively (Ichinose et al. (2008)).

Integrated sympathetic efferent output of the CNS:

$$
\begin{aligned}
f_{e f s}=\operatorname{Min}\left\{f_{e s, E}\right. & +\left(f_{e s, S}-f_{e s, E}\right) \cdot \exp \left[-k_{e s} \cdot\left(F_{a o r} \cdot f_{a f, a o r}+F_{c a r} \cdot f_{a f, c a r}+\Delta f\left(t_{m u s}\right)_{m e t}\right)\right] \\
& \left.+F_{v} \cdot f_{e f s, V}+\Delta f_{m e c}, \quad f_{e s, \max }\right\} .
\end{aligned}
$$


Jin, Liang and Liu, Journal of Biomechanical Science and Engineering, Vol.11, No.1 (2016)

Table B Parameters Values

\begin{tabular}{|c|c|c|c|c|c|c|c|}
\hline \multicolumn{2}{|c|}{ Metabovasodilation Model } & \multicolumn{2}{|c|}{ Set-point pressure } & \multicolumn{2}{|c|}{ Central Command Model } & \multicolumn{2}{|c|}{$\begin{array}{l}\text { Summation of the } \\
\text { baroreflex }\end{array}$} \\
\hline Parameters & Values & Parameters & Values & Parameters & Values & Parameters & Values \\
\hline$\Delta \mathrm{R}_{\mathrm{rat}, \min }$ & $\begin{array}{l}0.0[\mathrm{mmHg} \\
\left./ \mathrm{ml}^{3}\right]\end{array}$ & $\Delta p_{\text {exe }, \max }$ & $25.0[\mathrm{mmHg}]$ & $I_{\text {mus, } \max }$ & $\begin{array}{l}5.0[\mathrm{mmHg} \\
\left.\cdot \mathrm{ml} \cdot \mathrm{sec}^{-1}\right]\end{array}$ & $k_{m e c}$ & $\begin{array}{l}0.051[\mathrm{~Hz} \\
/ \mathrm{mmHg}]\end{array}$ \\
\hline$\Delta \mathrm{R}_{\text {rat,max }}$ & $\begin{array}{l}0.35[\mathrm{mmHg} \\
\left./ \mathrm{ml}^{3}\right]\end{array}$ & $\phi$ & $\begin{array}{l}1.8[\mathrm{mmHg} \\
\left.\cdot \mathrm{ml} \cdot \mathrm{sec}^{-1}\right]\end{array}$ & $\Delta f_{v a g, \max }$ & $2.8[\mathrm{~Hz}]$ & $\phi_{m e c}$ & $0.0[\mathrm{~Hz}]$ \\
\hline$D_{\text {rat }}$ & $12.0[\mathrm{sec}]$ & $m$ & 1.5 & $\tau_{\text {vag }}$ & $1.5[\mathrm{sec}]$ & $D_{\text {met }}$ & $22.0[\mathrm{sec}]$ \\
\hline$\gamma$ & $\begin{array}{l}1.0 \\
{[\mathrm{mmHg} \cdot} \\
\left.\mathrm{ml} \cdot \mathrm{sec}^{-1}\right]\end{array}$ & $p_{n}$ & $92.0[\mathrm{mmHg}]$ & & & $k_{m e t}$ & $\begin{array}{l}2.3[\mathrm{~Hz} \\
/ \mathrm{mmHg}]\end{array}$ \\
\hline$\omega$ & $0.03[\mathrm{ml}]$ & & & & & $\phi_{m e t}$ & $0.0[\mathrm{~Hz}]$ \\
\hline$\tau_{\text {rat }}$ & $22.0[\mathrm{sec}]$ & & & & & & \\
\hline$n$ & 2.8 & & & & & & \\
\hline
\end{tabular}

\section{References}

Akinpelu D, Treamill Stress Testing, Medscape (2015).

Bevegård, B. S., and Shepherd, J. T., Circulatory effects of stimulating the carotid arterial stretch receptors in man at rest and during exercise, Journal of Clinical Investigation, Vol. 45, No.1 (1966), pp.132-142.

Burattini, R., Borgdorff, P., and Westerhof, N., The baroreflex is counteracted by autoregulation, thereby preventing circulatory instabilit, Experimental physiology, Vol. 89, No.4 (2004), pp.397-405.

Donald, D. E., and Edis, A. J., Comparison of aortic and carotid baroreflexes in the dog, The Journal of physiology, Vol. 215, No.2 (1971), pp.521-538.

Gisolf, J., Immink, R. V., Van Lieshout, J. J., Stok, W. J., and Karemaker, J. M., Orthostatic blood pressure control before and after spaceflight, determined by time-domain baroreflex method, Journal of Applied Physiology, Vol.98, No.5 (2005), pp.1682-1690.

Guo, G. B., Thames, M. D., and Abboud, F. M., Differential baroreflex control of heart rate and vascular resistance in rabbits. Relative role of carotid, aortic, and cardiopulmonary baroreceptors, Circulation research, Vol. 50, No.4 (1982), pp.554-565.

Heldt, T., Shim, E. B., Kamm, R. D., and Mark, R. G., Computational modeling of cardiovascular response to orthostatic stress, Journal of applied physiology, Vol. 92, No.3 (2002), pp.1239-1254.

Ichinose M., and Nishiyasu, T., Muscle metaboreflex modulates the arterial baroreflex dynamic effects on peripheral vascular conductance in humans, Heart and Circulatory Physiology, Vol. 288, No.4 (2005), pp.1532-1538.

Ichinose, M., Saito, M., Fujii, N., Ogawa, T., Hayashi, K., Kondo, N., and Nishiyasu, T., Modulation of the control of muscle sympathetic nerve activity during incremental leg cycling, The Journal of physiology, Vol. 586, No.11 (2008), pp.2753-2766.

Kawada, T., Inagaki, M., Takaki, H., Sato, T., Shishido, T., Tatewaki, T., Yanagiya, Y., Sugimachi, M., and Sunagawa, K., Counteraction of aortic baroreflex to carotid sinus baroreflex in a neck suction model. Journal of Applied Physiology, Vol.89, No.5 (2000), pp.1979-1984.

Laughlin, M. H., Skeletal muscle blood flow capacity: role of muscle pump in exercise hyperemia, American Journal of Physiology-Heart and Circulatory Physiology, Vol. 253, No.5 (1987), pp.993-1004.

$\mathrm{Li}, \mathrm{X}$., and Bai, J., Computer simulation of the baroregulation in response to moderate dynamic exercise, Medical and Biological Engineering and Computing, Vol. 39, No.4 (2001), pp.480-487.

Liang, F. Y., and Liu, H., Simulation of Hemodynamic Responses to the Valsalva Maneuver: An integrative Computational Model of the Cardiovascular System and the Autonomic Nervous System, The Journal of Physiological Sciences, Vol.56, No.1 (2006), pp.45-65.

Magosso, E., and Ursino, M. Cardiovascular response to dynamic aerobic exercise: A mathematical model, Medical and Biological Engineering and Computing, Vol. 40, No.6 (2002), pp.660-674.

Melvin Keller, D., Fadel, P. J., Ogoh, S., Matthew Brothers, R., Hawkins, M., Olivencia - Yurvati, A., and Raven, P. B., 
Carotid baroreflex control of leg vasculature in exercising and non - exercising skeletal muscle in humans, The Journal of physiology, Vol. 561, No.1 (2004), pp.283-293.

Mohrman, D. E. and Heller, L. J., Cardiovascular Physiology (2006), p.1-2, Lange Physiology Series.

Norton, K. H., Boushel, R., Strange, S., Saltin, B., \& Raven, P. B., Resetting of the carotid arterial baroreflex during dynamic exercise in humans, Journal of Applied Physiology, Vol. 87, No.1 (1999), pp.332-338.

Olufsen, M. S., Ottesen, J. T., Tran, H. T., Ellwein, L. M., Lipsitz, L. A., and Novak, V., Blood pressure and blood flow variation during postural change from sitting to standing: model development and validation, Journal of Applied Physiology, Vol.99, No. 4 (2005), pp.1523-1537.

Papelier, Y., Escourrou, P., Gauthier, J. P., \& Rowell, L. B., Carotid baroreflex control of blood pressure and heart rate in men during dynamic exercise, Journal of Applied Physiology, Vol. 77, No.2 (1994), pp.502-506.

Potts, J. T., Shi, X. R., and Raven, P. B., Carotid baroreflex responsiveness during dynamic exercise in humans, American Journal of Physiology-Heart and Circulatory Physiology, Vol. 265, No.6 (1993), pp.1928-1938.

Radegran, G., and Saltin, B., Muscle blood flow at onset of dynamic exercise in humans, Heart and Circulatory Physiology, Vol. 274, No.1 (1998), pp.314-322.

Sheriff, D. D., Rowell, L. B., and Scher, A. M., Is rapid rise in vascular conductance at onset of dynamic exercise due to muscle pump?, American Journal of Physiology-Heart and Circulatory Physiology, Vol.265, No.4 (1993)., pp.1227-1234.

Sprangers, R. L. H., Wesseling, K. H., Imholz, A. L. T., Imholz, B. P. M., and Wieling W., Initial blood pressure fall on stand up and exercise explained by changes in total peripheral resistance, Journal of Applied Physiology, Vol.70, No.2 (1991), pp523-530.

Sun, Y., Beshara, M., Lucariello, R. J., \& Chiaramida, S. A., A comprehensive model for right-left heart interaction under the influence of pericardium and baroreflex, American Journal of Physiology-Heart and Circulatory Physiology, Vol. 272, No.3 (1997), pp.1499-1515.

Takahashi, T., Okada, A., Saitoh, T., Hayano, J., and Miyamoto, Y., Difference in human cardiovascular response between upright and supine recovery from upright cycle exercise, European journal of applied physiology, Vol. 81, No.3 (2000), pp.233-239.

Yamamoto, K., Kawada, T., Kamiya, A., Takaki, H., Sugimachi, M., and Sunagawa, K., Static interaction between muscle mechanoreflex and arterial baroreflex in determining efferent sympathetic nerve activity, American Journal of Physiology-Heart and Circulatory Physiology, Vol. 289, No.4 (2005), pp.1604-1609. 